\title{
Two Cases of Israeli Spotted Fever with Purpura Fulminans, Sharon District, Israel
}

\author{
Regev Cohen, Frida Babushkin, Maurice Shapiro, Martina Uda, \\ Yafit Atiya-Nasagi, Dar Klein, Talya Finn
}

\section{Medscape ACTIVITY}

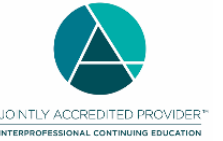

SIONAL CONTINUING EDUCaTion

In support of improving patient care, this activity has been planned and implemented by Medscape, LLC and Emerging Infectious Diseases. Medscape, LLC is jointly accredited by the Accreditation Council for Continuing Medical Education (ACCME), the Accreditation Council for Pharmacy Education (ACPE), and the American Nurses Credentialing Center (ANCC), to provide continuing education for the healthcare team.

Medscape, LLC designates this Journal-based CME activity for a maximum of 1.00 AMA PRA Category 1 Credit(s) ${ }^{\mathrm{TM}}$. Physicians should claim only the credit commensurate with the extent of their participation in the activity.

All other clinicians completing this activity will be issued a certificate of participation. To participate in this journal CME activity: (1) review the learning objectives and author disclosures; (2) study the education content; (3) take the post-test with a $75 \%$ minimum passing score and complete the evaluation at http://www.medscape.org/journal/eid; and (4) view/print certificate. For CME questions, see page 961.

Release date: April 12, 2018; Expiration date: April 12, 2019

\section{Learning Objectives}

Upon completion of this activity, participants will be able to:

1. Assess different subspecies of Rickettsia conorii in terms of their virulence

2. Evaluate common presenting symptoms and signs of Israel spotted fever

3. Analyze different laboratory findings in the current case series of Israel spotted fever

\section{CME Editor}

Thomas J. Gryczan, MS, Technical Writer/Editor, Emerging Infectious Diseases. Disclosure: Thomas J. Gryczan, MS, has disclosed no relevant financial relationships.

\section{CME Author}

Charles P. Vega, MD, FAAFP, Health Sciences Clinical Professor, University of California, Irvine, Department of Family Medicine; Associate Dean for Diversity and Inclusion, University of California, Irvine, School of Medicine; Executive Director, University of California, Irvine, Program in Medical Education for the Latino Community, Irvine, California. Disclosure: Charles $P$. Vega, MD, FAAFP, has disclosed the following relevant financial relationships: served as an advisor or consultant for Johnson \& Johnson Healthcare; served as a speaker or a member of a speakers bureau for Shire Pharmaceuticals.

\section{Authors}

Disclosures: Regev Cohen, MD; Frida Babushkin, MD; Maurice Shapiro, MD; Martina Uda, MD; Yafit Atiya-Nasagi, PhD; Dar Klein, MSc; and Talya Finn, MBBS, have disclosed no relevant financial relationships.

Author affiliations: The Ruth and Bruce Rappaport Faculty of Medicine, Technion, Haifa, Israel (R. Cohen); Laniado Hospital, Netanya, Israel (R. Cohen, F. Babushkin, M. Shapiro, M. Uda, T. Finn); The Israel Institute for Biological Research,

Ness-Ziona, Israel (Y. Atiya-Nasagi, D. Klein)

DOI: https://doi.org/10.3201/eid2405.171992
We report a series of 5 case-patients who had Israeli spotted fever, of whom 2 had purpura fulminans and died. Four case-patients were given a diagnosis on the basis of PCR of skin biopsy specimens 3-4 days after treatment with doxycycline; 1 case-patient was given a diagnosis on the basis of seroconversion. Rickettsia spp. from the 2 case-patients who died were sequenced and identified as Rickettsia 
conorii subsp. israelensis. Purpura fulminans has been described in association with $R$. rickettsii and $R$. indica, but rarely with $R$. conorii subsp. israelensis.

$R$ ickettsia conorii is the etiologic agent of Mediterranean spotted fever (MSF), which is considered one of the most severe and life-threatening rickettsial infections. Among the 4 strains of $R$. conorii ( $R$. conorii subsp. conorii, $R$. conorii subsp. caspia, $R$. conorii subsp. indica, and $R$. conorii subsp. israelensis), $R$. conorii subsp. israelensis, which causes Israeli spotted fever (ISF), is believed to be the most virulent strain and shows a case-fatality rate of up to $32.3 \%$ in hospitalized patients (1). R. conorii is endemic to Israel (2). However, in recent years, Rickettsia spp. other than $R$. conorii have been identified and reported from Isra$\mathrm{el}$, including $R$. africae, $R$. massiliae, and $R$. sibirica $(3,4)$.

Within the $R$. conorii group, there are clinical and virulence differences. For example, infections with $R$. conorii subsp. israelensis have higher case-fatality rates than infections with the $R$. conorii Malish strain $(29 \%$ vs. $13 \%)$, and $R$. conorii subsp. israelensis is rarely associated with an eschar at the site of a tick bite $(5,6)$. ISF has also been reported from other Mediterranean countries, including Portugal (7), Italy and Sicily (6), and Tunisia (8). R. conorii subsp. conorii that cause MSF are also endemic to Europe, Asia, and Africa.

ISF usually manifests as fever with a maculopapular rash, usually involving the palms of the hands and the soles of the feet. Patients who die from ISF typically have multiorgan failure, acute renal or hepatic failure, and acute encephalitis; all are attributed to the affinity of the Rickettsia spp. to endothelial cells with resultant vasculitis. The typical maculopapular rash might transform to become hemorrhagic with discrete purpurial lesions, but true manifestation of ISF purpura fulminans is not frequently reported in Israel or from other countries.

Because serologic cross-reactivity occurs across the spotted fever group (SFG) rickettsiae (9) and the primary means of diagnosis is through serum antibody assays, accurate distinction between different subspecies requires identification of the actual infecting bacterium. This cross-reactivity, overlap in geographic distributions, and the different clinical severities highlight the need to better differentiate between these rickettsial species. A correct diagnosis is critical for predicting the pathologic complications that would arise because of infection (10).

We report a case series of 5 patients with ISF from the same geographic area (Sharon District in Israel) (Tables 1, 2). Four of these case-patients were detected during AprilMay 2017. Two case-patients had purpura fulminans and both died. These cases were a part of a national outbreak of SFG rickettsiosis.

\section{Case-Patient 1}

A 75-year-old woman with a history of hypertension, a tourist from Ukraine who had been staying with her daughter in Netanya for the previous 3 months, came to an emergency department in October 2016 with 6 days of fever, weakness, and anorexia. She had been exposed to 3 dogs at the house of her daughter but had no recollection of tick exposure. At admission, she was coherent but in a state of septic shock; she was hypotensive and had a disseminated, fern-leaf pattern, purpural rash over her body, including the face (Figure 1). Laboratory findings included leukocytosis, severe thrombocytopenia $(23,000$ platelets $/ \mu \mathrm{L})$, hyponatremia (sodium level $126 \mathrm{mmol} / \mathrm{L}$ ), acute renal failure, increased levels of liver enzymes and creatine kinase (CK), and disseminated intravascular coagulation (Table 1).

She was admitted to the intensive care unit (ICU) and given broad-spectrum antimicrobial drug therapy, including intravenous doxycycline. During the next 2 days, multiorgan failure, severe jaundice, liver failure, and acute lung injury developed, and the rash became bullous with clear serous fluid (Figure 2). A skin biopsy specimen from a hemorrhagic lesion was obtained and tested by PCR. The result was positive for $R$. conorii subsp. israelensis. Serologic analysis for rickettsia at the time of admission (day 9 after disease onset) showed no reactivity, but another sample obtained 10 days later was positive (IgG titer 1:800) for SFG rickettsiae.

\begin{tabular}{|c|c|c|c|c|c|c|}
\hline Parameter & Reference range & Case-patient 1 & Case-patient 2 & Case-patient 3 & Case-patient 4 & Case-patient 5 \\
\hline Leukocytes, cells/ $\mu \mathrm{L}$ & $4,000-11,000$ & 10,700 & 7,500 & 5,000 & 7,700 & 8,600 \\
\hline PMNs, $\%$ & $40-75$ & 87 & 86 & 81 & 85 & 91 \\
\hline Platelets $/ \mu \mathrm{L}$ & $150,000-400,000$ & 37,000 & 73,000 & 91,000 & 75,000 & 139,000 \\
\hline Sodium, mmol/L & $136-145$ & 126 & 123 & 130 & 133 & 127 \\
\hline Total bilirubin, mg/dL & $0.3-1.4$ & 2.5 & 0.4 & 0.9 & 0.7 & 0.8 \\
\hline AST, IU/L & $0-32$ & 106 & 184 & 167 & 65 & 69 \\
\hline ALT, IU/L & $0-33$ & 51 & 154 & 139 & 68 & 66 \\
\hline GGT, IU/L & $0-40$ & 50 & 103 & 62 & 26 & 106 \\
\hline LDH, IU/L & $240-480$ & 1044 & 875 & 1195 & 466 & 536 \\
\hline Creatinine, mg/dL & $0.5-0.9$ & 1.7 & 1.4 & 1.0 & 1.4 & 1.0 \\
\hline Creatine kinase, IU/L & $39-190$ & 298 & 1933 & 152 & 159 & 100 \\
\hline C-reactive protein, $\mathrm{mg} / \mathrm{L}$ & $0-5$ & 122 & 350 & 164 & 88 & 240 \\
\hline
\end{tabular}


Table 2. Clinical characteristics of 5 case-patients with Israeli spotted fever, Sharon District, Israel*

\begin{tabular}{|c|c|c|c|c|c|}
\hline Characteristic & Case-patient 1 & Case-patient 2 & Case-patient 3 & Case-patient 4 & Case-patient 5 \\
\hline Age, y/sex & $75 / \mathrm{F}$ & $51 / \mathrm{F}$ & $38 / \mathrm{M}$ & 48/M & $45 / \mathrm{M}$ \\
\hline Concurrent condition & Hypertension & $\begin{array}{l}\text { Mental retardation, } \\
\text { diabetes mellitus }\end{array}$ & None & $\begin{array}{l}\text { Alcoholism, HCV } \\
\text { carrier, IVDU }\end{array}$ & $\begin{array}{l}\text { Nephrolithiasis, } \\
\text { psoriasis }\end{array}$ \\
\hline \multicolumn{6}{|l|}{ Signs/symptoms at hospitalization } \\
\hline Fever & Yes & Yes & Yes & Yes & Yes \\
\hline Rash & $\begin{array}{l}\text { Purpura } \\
\text { fulminans }\end{array}$ & $\begin{array}{l}\text { Maculopapular, then } \\
\text { purpura fulminans }\end{array}$ & Maculopapular & Maculopapular & Maculopapular \\
\hline Day of rash from disease onset & 6 & 6 & 4 & 4 & 3 \\
\hline Hypotension & Yes & Yes & No & No & No \\
\hline Multiorgan failure & Yes & Yes & No & No & No \\
\hline Eschar & No & No & No & No & No \\
\hline Headache & No & Unknown & Yes & No & No \\
\hline Confusion & No & No & No & No & No \\
\hline Myalgia & Yes & Unknown & Yes & No & No \\
\hline \multicolumn{6}{|l|}{ Epidemiologic factor } \\
\hline Animal exposure & Dog & Unknown & Dog & None & Dog \\
\hline Tick exposure & No & No & No & No & No \\
\hline \multicolumn{6}{|l|}{ Serologic result, day/result } \\
\hline First sample & 9/nonreactive & $\begin{array}{l}6 / \lg M \text { titer }>100, \lg G \\
\text { titer borderline }\end{array}$ & 6/nonreactive & 6/nonreactive & 5/nonreactive \\
\hline Second sample & $\begin{array}{c}\text { 19/IgM titer } \\
\text { nonreactive, IgG } \\
\text { titer } 1: 800 \dagger\end{array}$ & NR & NR & NR & $\begin{array}{c}\text { 19/lgM titer } \\
>1: 100, \text { IgG titer } \\
1: 1,600 \dagger\end{array}$ \\
\hline $\begin{array}{l}\text { PCR result, whole } \\
\text { blood/doxycycline treatment day }\end{array}$ & NR & $+/ 3$ & NR & NR & -15 \\
\hline $\begin{array}{l}\text { PCR result, skin biopsy } \\
\text { specimen/doxycycline treatment day }\end{array}$ & $+/ 4$ & $+/ 3$ & $+/ 4$ & $+/ 3$ & $-/ 3$ \\
\hline \multicolumn{6}{|l|}{ Doxycycline treatment } \\
\hline Day of illness & 6 & 5 & 5 & 5 & 4 \\
\hline Day of hospitalization & 0 & 2 & 0 & 1 & 0 \\
\hline Length of hospitalization, $\mathrm{d}$ & 14 & 20 & 7 & 4 & 8 \\
\hline Outcome & Died & Died & Survived & Survived & Survived \\
\hline
\end{tabular}

The skin lesions became dusky, necrosis appeared at the extremities (Figure 3), and there was extensive desquamation. The patient died of candidemia after 14 days in ICU.

\section{Case-Patient 2}

A 51-year-old woman with diabetes, who was mentally retarded and lived in a nursing home was hospitalized in an internal medicine ward on May 2017 with fever of no obvious source. She had no known exposure to dogs or ticks at the nursing home. The case-patient was hypotensive at admission, and laboratory results included a standard leukocyte count, thrombocytopenia $(73,000$ platelets $/ \mu \mathrm{L})$, hyponatremia, acute renal failure, increased levels of CK $(1,933 \mathrm{IU} / \mathrm{L})$ and liver enzymes, and a C-reactive protein level of $350 \mathrm{mg} / \mathrm{L}$ (Table 1). She was treated empirically with ceftriaxone.

On the third day of hospitalization, an infectious diseases consultation was performed, and intravenous doxycycline was added to the treatment regimen. She was transferred to the ICU unit, and the next day a maculopapular rash developed, which included the palms and soles; the rash rapidly became confluent and bullous, and skin necrosis followed.

PCR results for a whole blood sample and a skin biopsy specimen were positive for $R$. conorii subsp. israelensis.
Serologic analysis on day 6 of the fever showed an IgM titer $>1: 100$ and a borderline IgG titer of 1:100 for rickettsia.

Despite treatment with doxycycline, multiorgan failure developed and the patient died of Pseudomonas aeruginosa bacteremia related to central line infection 3 weeks after admission.

\section{Case-Patients 3-5}

Three other case-patients were admitted to Laniado Hospital (Netanya, Israel) during April-May 2017 for fever and a maculopapular rash involving the palms that appeared on the third or fourth day after fever onset. Two patients had exposure to dogs, but none reported exposure to ticks. One patient had alcoholism, but he had a benign illness course. The diagnosis for 2 patients was made by using PCR for skin biopsy specimens, and the diagnosis for the third patient was made by using seroconversion. Skin biopsy PCR results were positive despite 3-4 days of doxycycline treatment.

\section{Methods}

\section{Serologic Analysis}

Serologic diagnosis was made by using indirect immunofluorescence assays. Serum samples were obtained from 5 


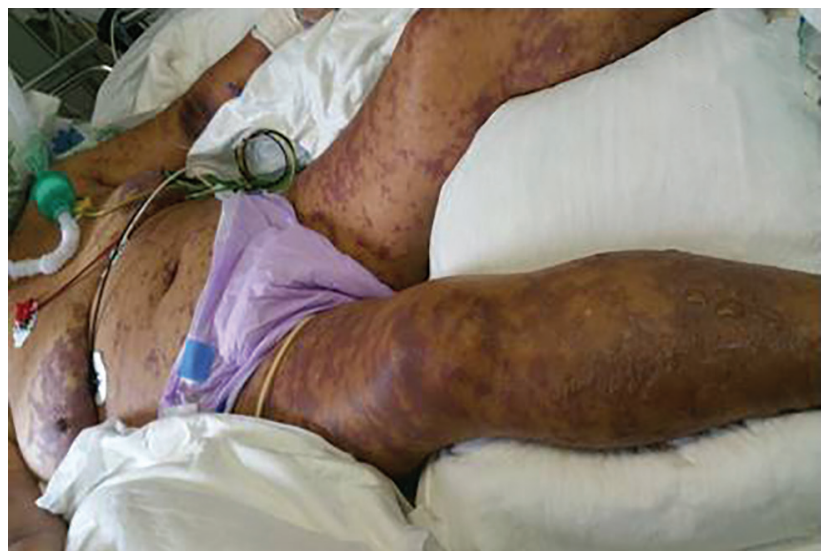

Figure 1. Case-patient 1, a 75-year-old woman with Israeli spotted fever and purpura fulminans, Sharon District, Israel, 3 days after hospitalization. Diffuse fern-leaf pattern of purpura and newly formed bulla on the legs are shown.

patients and tested for antibodies against $R$. conorii and $R$. typhi as described (11).

\section{DNA Extraction and PCR Detection}

We extracted DNA from skin biopsy or whole blood specimens from 4 patients by using the QIAamp Mini Kit (QIAGEN, Valencia, CA, USA) according to the manufacturer's instructions. We tested samples for SFG rickettsiae by using nested PCR to amplify a fragment of the $17-\mathrm{kDa}$ protein antigen gene as described (12). For further identification, we performed an additional PCR by using primers derived from consensus sequences of the rickettsial outer membrane protein A gene (213F 5'-AATCAATATTGGAGCCGGTAA-3' and 667R 5'-ATTTGCATCAATCGTATAAGTAGC-3'). We analyzed sequences by using BLAST (https://blast.ncbi.nlm.nih.gov/Blast.cgi). This fragment was identical to that of the Israeli tick typhus rickettsial outer membrane protein A gene (GenBank accession no. U83441.1).

\section{Discussion}

SFG rickettsial disease is endemic to Israel and might be related to deaths of infected patients. However, manifestation of ISF as primary purpura fulminans has been described only rarely. Thus, we report patients with rickettsial disease and purpura fulminans.

Among various species of SFG rickettsiae, the types known to be associated with patient deaths are $R$. rickettsii (agent of Rocky Mountain spotted fever); $R$. conorii of the subtypes conorii, israelensis, and indica; and rarely $R$. australis (13). Although patient deaths are associated with vasculitis and multiorgan failure, purpura fulminans as part of the clinical presentation is a feature seen only for a small subset of the species, namely $R$. rickettsii, $R$. australis (14), and probably $R$. conorii subsp. indica. R. conorii

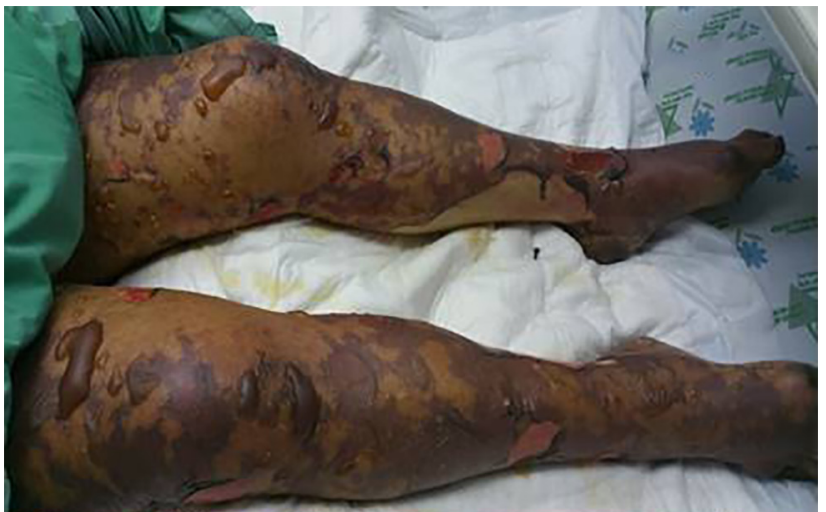

Figure 2. Case-patient 1, a 75-year-old woman with Israeli spotted fever and purpura fulminans, Sharon District, Israel, 7 days after hospitalization. A rash on the legs that had become bullous and contained clear serous fluid is shown.

subsp. indica was reported in several case series from the Indian subcontinent as a cause of fern-leaf pattern purpura, although the specific rickettsial subtype was infrequently identified in these studies (15-20).

Purpura fulminans is not a typical feature of ISF in reviews and in case series from Israel and other countries to which $R$. conorii subsp. israelensis is endemic $(5,6,21)$. Although there are reports of deaths of patients in Israel who had a clinical picture suspected initially to be meningococcemia, fern-leaf purpura, as for 2 of our case-patients, was not part of the clinical descriptions (22). We have found only 1 case report of $R$. conorii subsp. israelensis infection and purpura fulminans (23). We report 2 additional cases.

The type of severe skin damage in the 2 case-patients we report is not the natural history of fatal spotted fever cases because it has not been described in other patients dying of ISF. The severe and confluent skin lesions undoubtedly contributed to bloodstream infections, leading to death

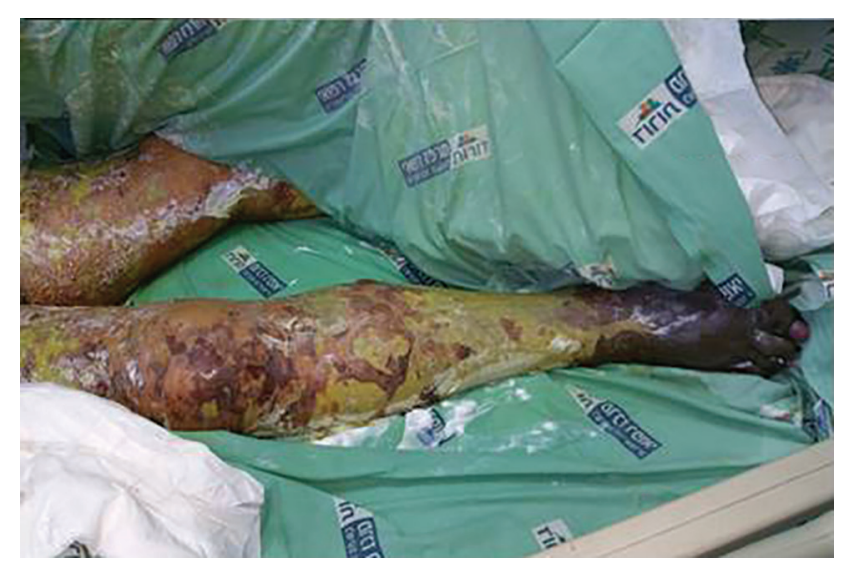

Figure 3. Case-patient 1, a 75-year-old woman with Israeli spotted fever and purpura fulminans, Sharon District, Israel, 14 days after hospitalization. Dusky skin lesions on the legs, necrosis at the extremities, and extensive desquamation are shown. 
in both cases. Because the differential diagnosis of purpura fulminans in Israel usually includes microorganisms other than rickettsiae, this deadly, unreported, unique, and potentially misleading manifestation of ISF must be recognized and treated in a timely fashion.

The clinical presentation of case-patients in this case series was typical of ISF and compatible with other case series of ISF (Table 2) $(5,24)$. The reasons why 2 of these patients died and the other 3 patients had relatively mild disease is not entirely clear. Both case-patients who died were older; had hypotension, purpura fulminans, multiorgan failure, and acute renal failure; and showed increased levels of CK. Delay in doxycycline administration was evident for case-patient 1 (day 6 after illness onset) because she was hospitalized at a later time. A delay in doxycycline administration during medical observation occurred for case-patient 2, although treatment was given on day 5 after disease onset, which was similar to the timing for the 3 case-patients who survived.

We sequenced rickettsiae isolated from the 2 casepatients who died; both were positive for $R$. conorii subsp. israelensis. However, we did not perform sequencing for isolates from the other 2 case-patients who were positive by PCR for the SFG rickettsiae. These findings suggest that disease severity might be related to the specific subtype, and not to host factors, but further research is required. Alcoholism has been reported a risk factor for fatal disease (5), but the patient in this case series who had alcoholism had a benign course of illness.

With regards to epidemiologic, clinical, and laboratory findings, as expected, none of our patients had an eschar or known exposure to ticks. One patient had diarrhea, but 2 patients had constipation. All 5 case-patients had rash and laboratory abnormalities typical of ISF (Table 1), as described (24).

As previously reported $(6,24)$, serologic analysis was not useful in the diagnosis of ISF because results were nonreactive for all case-patients early in the disease course and positive for 2 case-patients tested during the convalescent period (19 days after disease onset). In contrast, whole blood and skin biopsy specimen PCRs were helpful in obtaining a diagnosis. PCR results for skin biopsy specimens were positive for 4 of the 5 case-patients even though these PCRs were performed for 4 patients 3-4 days after doxycycline therapy was begun. The 1 case-patient with a negative PCR result for a skin biopsy specimen also had no inflammatory changes by histologic analysis, Thus, the negative PCR result for this patient probably represented a sampling error and not a false-negative result.

PCR for whole blood was performed for 2 patients: case-patient 2 showed a positive result after 3 days of doxycycline therapy, and case-patient 5 showed a negative result after 5 days of doxycycline therapy. Reduced sensitivity of whole blood PCR after beginning antimicrobial drug therapy has been reported (24). For the 4 casepatients with positive PCR results for skin biopsy specimens, the pathology report did not indicate a diagnosis of rickettsiosis, describing either intravascular coagulation and no vasculitis for the 2 patients who died and perivascular minimal lymphocytic infiltration not consistent with rickettsiosis for the other 2 patients.

Our cluster was a part of an outbreak throughout Israel during the spring and summer of 2017. This outbreak included deaths of 2 healthy young persons. One of these persons was a 15 -year-old boy from the Sharon District. During July 2017, the Israeli Ministry of Health reported on an increased incidence of spotted fever cases ( 11 cases during January-May 2017); the yearly average is 6 cases (25).

In summary, during the spring and summer of 2017, there was an increased incidence of spotted fever in Israel, causing the deaths of 4 patients. Three of these patients acquired the disease in the Sharon District, and 2 were seen in our facility. Two of the case-patients who died were confirmed by sequencing to be infected with $R$. conorii subsp. israelensis; both of these patients had purpura fulminans and multiorgan failure. Purpura fulminans should increase the suspicion of ISF, along with other better-described pathogens, such as Neisseria meningitidis, and diagnostic and therapeutic measures must be taken urgently.

PCR of skin biopsy specimens is useful in providing a rapid and accurate diagnosis, even if performed 3-4 days after beginning of treatment, and PCR of whole blood might also show positive results during antimicrobial drug treatment early in the disease course. Histopathologic results for skin lesions might be inaccurate and cause a delay in diagnosis or an erroneous diagnosis. Features of R. conorii subsp. israelensis should be further explored primarily by performing genetic sequencing of all isolates from cases of spotted fever diagnosed in Israel to distinguish it from other spotted fever rickettsiae or $R$. conorii subsp. conorii that might cause a less aggressive disease.

\section{About the Author}

Dr. Cohen is head of the Infectious Diseases Unit, Sanz Medical Center, Laniado Hospital, Netanya, Israel. His research interests are vectorborne diseases, zoonoses, and healthcareassociated infections.

\section{References}

1. de Sousa R, Nóbrega SD, Bacellar F, Torgal J. Mediterranean spotted fever in Portugal: risk factors for fatal outcome in 105 hospitalized patients. Ann N Y Acad Sci. 2003;990:285-94. http://dx.doi.org/10.1111/j.1749-6632.2003.tb07378.x

2. Mumcuoglu KY, Keysary A, Gilead L. Mediterranean spotted fever in Israel: a tick-borne disease. Isr Med Assoc J. 2002;4:44-9.

3. Waner T, Keysary A, Eremeeva ME, Din AB, Mumcuoglu KY, King R, et al. Rickettsia africae and Candidatus Rickettsia 
barbariae in ticks in Israel. Am J Trop Med Hyg. 2014;90:920-2. http://dx.doi.org/10.4269/ajtmh.13-0697

4. Harrus S, Perlman-Avrahami A, Mumcuoglu KY, Morick D, Baneth G. Molecular detection of Rickettsia massiliae, Rickettsia sibirica mongolitimonae and Rickettsia conorii israelensis in ticks from Israel. Clin Microbiol Infect. 2011;17:176-80. http://dx.doi.org/10.1111/j.1469-0691.2010.03224.x

5. Sousa R, França A, Dória Nòbrega S, Belo A, Amaro M, Abreu T, et al. Host- and microbe-related risk factors for and pathophysiology of fatal Rickettsia conorii infection in Portuguese patients. J Infect Dis. 2008;198:576-85. http://dx.doi.org/10.1086/590211

6. Colomba C, Trizzino M, Giammanco A, Bonura C, Di Bona D, Tolomeo M, et al. Israeli spotted fever in Sicily: description of two cases and minireview. Int J Infect Dis. 2017;61:7-12. http://dx.doi.org/10.1016/j.ijid.2017.04.003

7. Chai JT, Eremeeva ME, Borland CD, Karas JA. Fatal Israeli spotted fever in a UK traveler to South Portugal. J Travel Med. 2008; 15:122-3. http://dx.doi.org/10.1111/j.1708-8305.2007.00179.x

8. Znazen A, Hammami B, Lahiani D, Ben Jemaa M, Hammami A. Israeli spotted fever, Tunisia. Emerg Infect Dis. 2011;17:1328-30. http://dx.doi.org/10.3201/eid1707.101648

9. Maia C, Ferreira A, Nunes M, Vieira ML, Campino L, Cardoso L. Molecular detection of bacterial and parasitic pathogens in hard ticks from Portugal. Ticks Tick Borne Dis. 2014;5:409-14. http://dx.doi.org/10.1016/j.ttbdis.2014.01.009

10. Bechelli J, Smalley C, Milhano N, Walker DH, Fang R. Rickettsia massiliae and Rickettsia conorii Israeli spotted fever strain differentially regulate endothelial cell responses. PLoS One. 2015;10:e0138830. http://dx.doi.org/10.1371/journal.pone.0138830

11. Keysary A, Strenger C. Use of enzyme-linked immunosorbent assay techniques with cross-reacting human sera in diagnosis of murine typhus and spotted fever. J Clin Microbiol. 1997;35:1034-5.

12. Leitner M, Yitzhaki S, Rzotkiewicz S, Keysary A. Polymerase chain reaction-based diagnosis of Mediterranean spotted fever in serum and tissue samples. Am J Trop Med Hyg. 2002;67:166-9. http://dx.doi.org/10.4269/ajtmh.2002.67.166

13. Parola P, Paddock CD, Socolovschi C, Labruna MB, Mediannikov O, Kernif T, et al. Update on tick-borne rickettsioses around the world: a geographic approach. Clin Microbiol Rev. 2013;26:657-702. http://dx.doi.org/10.1128/CMR.00032-13

14. McBride WJ, Hanson JP, Miller R, Wenck D. Severe spotted fever group rickettsiosis, Australia. Emerg Infect Dis. 2007;13:1742-4. http://dx.doi.org/10.3201/eid1311.070099

15. Kundavaram A, Francis NR, Jude AP, Varghese GN. Acute infectious purpura fulminans due to probable spotted fever. J Postgrad Med. 2014;60:198-9. http://dx.doi.org/10.4103/0022-3859.132345
16. Weerakoon K, Kularatne SA, Rajapakse J, Adikari S, Waduge R. Cutaneous manifestations of spotted fever rickettsial infections in the Central Province of Sri Lanka: a descriptive study. PLoS Negl Trop Dis. 2014;8:e3179. http://dx.doi.org/10.1371/ journal.pntd.0003179

17. Katoch S, Kallappa R, Shamanur MB, Gandhi S. Purpura fulminans secondary to rickettsial infections: a case series. Indian Dermatol Online J. 2016;7:24-8. http://dx.doi.org/ 10.4103/2229-5178.174324

18. Luke N, Munasinghe H, Balasooriya L, Premaratna R. Widespread subcutaneous necrosis in spotted fever group rickettsioses from the coastal belt of Sri Lanka: a case report. BMC Infect Dis. 2017;17:278. http://dx.doi.org/10.1186/ s12879-017-2375-Z

19. Tirumala S, Behera B, Jawalkar S, Mishra PK, Patalay PV, Ayyagari S, et al. Indian tick typhus presenting as purpura fulminans. Indian J Crit Care Med. 2014;18:476-8. http://dx.doi.org/10.4103/0972-5229.136081

20. Joshi HS, Thomas M, Warrier A, Kumar S. Gangrene in cases of spotted fever: a report of three cases. BMJ Case Rep. 2012 Dec 18;2012:pii:bcr2012007295.

21. Keysary A, Potasman I, Itzhaki A, Finkelstein R, Yitzhaki S, Strenger C, et al. Clusters of Mediterranean spotted fever in Israel. Vector Borne Zoonotic Dis. 2007;7:143-6. http://dx.doi.org/10.1089/vbz.2006.0563

22. Yagupsky P, Wolach B. Fatal Israeli spotted fever in children. Clin Infect Dis. 1993;17:850-3. http://dx.doi.org/10.1093/ clinids/17.5.850

23. Weinberger M, Keysary A, Sandbank J, Zaidenstein R, Itzhaki A, Strenger C, et al. Fatal Rickettsia conorii subsp. israelensis infection, Israel. Emerg Infect Dis. 2008;14:821-4. http://dx.doi.org/10.3201/eid1405.071278

24. Ergas D, Sthoeger ZM, Keysary A, Strenger C, Leitner M, Zimhony O. Early diagnosis of severe Mediterranean spotted fever cases by nested-PCR detecting spotted fever Rickettsiae 17-kD common antigen gene. Scand J Infect Dis. 2008;40:965-7. http://dx.doi.org/10.1080/00365540802400584

25. Israel Center for Disease Control. Ministry of Health. Publication files, infectious diseases, notifiable infectious diseases in Israel, 2012 [cited 2018 Jan 19]. https://www.health.gov.il/ English/News_and_Events/Spokespersons_Messages/ Pages/05072017_2.aspx

Address for correspondence: Regev Cohen, Infectious Diseases Unit, Sanz Medical Center, Laniado Hospital, 16 Divrei Haim St, Kiryat Sanz, 42150, Netanya, Israel; email: regevc@laniado.org.il 